\title{
COMMENT
}

\section{Unintended consequences of restrictive visitation policies during the COVID-19 pandemic: implications for hospitalized children}

\author{
Jean L. Raphael ${ }^{1}$, Woodie Kessel ${ }^{2}$ and Mona Patel ${ }^{3}$ \\ Pediatric Research (2021) 89:1333-1335; https://doi.org/10.1038/s41390-021-01439-0
}

The COVID-19 pandemic has resulted in devastating consequences worldwide with over 2,000,000 deaths. Although COVID-19 demonstrates less morbidity and mortality among children, ${ }^{1}$ it has dramatically altered the health-care experience for children and families. This is particularly true for those cared for in inpatient settings. The competing priorities of safeguarding families and health-care personnel from a serious infection, stewardship of limited resources, ensuring family-centered care (FCC), and carrying out end-of-life care have led to tensions in how to effectively implement and execute necessary restrictive visitation policies. $^{2}$ While the Centers for Disease Control and Prevention (CDC) provides broad guidelines to health-care facilities on the management of visitors, hospitals must determine how to implement such guidelines. ${ }^{3}$ Many children's hospitals have instituted one-visitor policies with varying levels of restriction, such as allowing one parent at the bedside at any time or requiring families to choose a single parent to be allowed in the hospital for the entirety of the inpatient stay. Such visitation policies can have significant untoward consequences.

In this issue, Van Driest et al. ${ }^{4}$ explored the consent rate for an observational study conducted in the early months of the COVID19 pandemic after the implementation of a one-parent/visitor policy at an academic children's hospital. They calculated the consent rate and enrollment rate for historical periods, the time immediately preceding the one-visitor policy, and during the onevisitor policy. The authors found a statistically significant reduction in the consent rate for the observational study in the early months of the COVID-19 pandemic and during the implementation of the one-visitor policy. In this commentary, we focus on the effects of restrictive visitation policies and strategies to mitigate against adverse impacts on children and families, both in research and the clinical setting.

\section{RESEARCH IMPLICATIONS}

Researchers must consider the myriad of unintended consequences from restrictive visitation policies on children and families during a time of heightened anxiety related to the vulnerability and lethality of COVID-19 during the pandemic. Parents who feel isolated or distressed from being alone in the hospital during a pandemic may be less willing to participate in research. As another example, institutional review boards (IRBs) often require both parents to sign consent for important therapeutic trials in children or if studies are conducted on the fetus. If only one parent is allowed to visit and telephone consent is not allowed for the second parent, some children may not be able to participate in clinical trials. Van Driest et al. highlight the long-term consequences of the one-visitor policy on pediatric research identifying under enrollment, reduced sample sizes, and underrepresentation of key populations. Future pediatric research must adequately account for one-visitor policies in consent and enrollment procedures. ${ }^{5}$

\section{IMPACT ON FCC}

In addition to the conduct of research, COVID-19-related restrictive visitation policies have impacted how families and providers navigate the FCC model in the inpatient setting. In the FCC approach, health-care professionals work in partnership with families to facilitate parent-child closeness, open exchange of information, and shared decision-making regarding the child's health-care management. ${ }^{2,6}$ Endorsed by the American Academy of Pediatrics, the FCC has been demonstrated to improve outcomes for patients, families, and health-care providers while decreasing health-care expenditures. ${ }^{2,6}$ By their very nature, restrictive visitation policies limit the extent to which families and health-care providers in hospitals can engage in FCC. The consequences may be especially deleterious in settings such as the neonatal intensive care unit (NICU) where 24-h parental presence with extended intimate contact of the newborn has both physiological and psychological benefits to the newborn and parents. ${ }^{7}$ A recent study by Darcy Mahoney et al. ${ }^{8}$ assessed the impact of restrictions on parental presence in the NICU before and during the COVID-19 pandemic. The cross-sectional survey of global NICUs identified a variety of visitation policies among 130 NICUs worldwide that restricted parental presence during the COVID-19 pandemic. Of these NICUs, $85 \%$ only allowed one parent at the bedside at any time, with a subset $(25 \%)$ requiring families to choose a single parent to be allowed into the NICU for the entire hospitalization. Approximately $5 \%$ of NICUs excluded all parental presence. Such policies may have dramatic effects on kangaroo care or breastfeeding rates. This study also demonstrated dramatic shifts in parental engagement representative of FCC. The number of NICUs allowing 24-h parental presence decreased from 83 to $53 \%$ and parental participation in NICU rounds decreased from 71 to $32 \%$. As health-care systems adapt

\footnotetext{
${ }^{1}$ Center for Child Health Policy and Advocacy, Baylor College of Medicine, Houston, TX, USA; ${ }^{2}$ Department of Pediatrics, Geisel School of Medicine, Dartmouth College, Hanover, $\mathrm{NH}$, USA and ${ }^{3}$ Children's Hospital Los Angeles, Department of Pediatrics, Keck School of Medicine of USC, Los Angeles, CA, USA Correspondence: Jean L. Raphael (Raphael@bcm.edu)
}

Received: 16 January 2021 Accepted: 25 January 2021

Published online: 2 March 2021 
their visitation policies to prevent the spread of COVID-19, they may unintentionally amplify the underlying stress and anxiety of parents about their children's health during the pandemic, and impact engagement of families in supportive, shared decisionmaking with the health-care team.

\section{EXACERBATION OF HEALTH INEQUITIES}

Restrictive visitation policies in pediatric settings also have the potential to inadvertently exacerbate existing health-care inequities. Parents may weigh their physical presence in the hospital to engage in care and support of their child versus competing priorities such as work or caretaking of other children, which is especially challenging for marginalized populations during this global pandemic. If they choose to stay in the hospital, they may feel social isolation in combination with the stress of an ill child, without having shared family support during this time of crisis. If they remain at home, they forego the benefits of FCC and may feel the guilt of leaving a child alone in the hospital during a pandemic. They may also be subject to staff perceptions of being disengaged in their child's care, which further contributes to implicit bias already experienced by people of color in medical settings. ${ }^{9}$ For economically vulnerable populations, the weight of these decisions may be compounded by a lack of social supports and other child caretakers in addition to jobs with limited flexibility.

Vulnerable populations may also suffer if hospital's policies are not adequately informed by ethical principles, stakeholder engagement, accessibility, transparency, and acknowledgment of diversity and equity of the populations served. In a study of COVID-19 visitor policies in Michigan, Weiner et al. ${ }^{10}$ found that most policies lacked such elements, including stated ethical rationales and stakeholder engagement prior to implementation. Furthermore, many failed to delineate caregiver visitation exception request processes. Without such transparency, numerous institutional factors may lead to enforcement variation, and, consequently, confusion and inequity in visitation access and fair appeals processes. The consequence may be an amplification of already existing disparities as vulnerable populations may be less well-positioned to advocate for themselves. Inequities may also be engendered by the specification of which visitors are permitted, such as parents or immediate family. These assumptions of family structure may inadvertently exclude individuals important to the patient or those who have some level of decisional authority to the patient's care. ${ }^{10}$

\section{REDUCTION IN HEALTH-CARE UTILIZATION}

There is also concern that visitor restriction policies may lead families to delay or defer care that requires a hospitalization. Those facing the added burden of restrictions on parental presence may avoid or defer medical procedures or surgeries that will ultimately improve their child's health or well-being. Surgery, in normal times, is an anxiety-inducing experience for children and families. Family members and other social supports serve important roles in the preoperative decision-making, ensuring FCC throughout the hospitalization during recovery processes and in discharge planning. Visitor restrictions, made to stem the harm from the COVID-19 pandemic, may also negatively influence the postoperative experience. A recent study of adult postoperative experiences during COVID-19 by Zeh and Coworkers $^{11}$ highlighted the psychosocial impacts of restrictive visitation policies. Patients under no-visitor policies were more likely to be dissatisfied with their hospital experience. A lack of visitors negatively affected patient's psychosocial well-being. Patients under no-visitor policies were less likely to have their preferences adequately addressed at discharge. While no-visitor policies are unlikely to exist in pediatrics, even the limitation to one-visitor may adversely impact perioperative experiences. With consideration of these visitation restrictions, parents may opt to delay surgeries. This may impact the health of their children as well as the financial viability of hospitals already under financial strain during the pandemic.

\section{MITIGATING THE CHALLENGES OF VISITOR RESTRICTIONS DURING THE COVID-19 PANDEMIC}

As public health leaders, clinical experts, ethicists, and hospitals debate the merits of current visitation policies to stem the transmission of COVID-19, urgent efforts are needed to support families and mitigate against the unintended consequences of such restrictions on research, patient experience, and health equity. Researchers must modify policies and procedures while proactively working with IRBs to overcome challenges. Assuring that visitation policies are carried out in an ethically rigorous manner with transparency (e.g., clear appeals process), communication (e.g., public-facing website), and patient-centeredness (e.g., support mechanisms) is also essential., ${ }^{2,7,10}$ This requires periodic modification of policies informed by epidemiological data, protective equipment availability, and stakeholder engagement. Specific engagement of vulnerable at-risk populations is critical in order to prevent exacerbation of health inequities. In addition to revising policies, hospitals must develop comprehensive measures to support parents during this or any infectious disease threat. Upon admission, inpatient clinical teams could provide families with a communication plan outlining their policy and specific measures by which they can experience FCC regardless of whether they are at the bedside or home. Parents should have a clear understanding of technologies, platforms, and interpreter services available to the patient and family for communication. Social work providers should be involved early in the hospitalization to describe available psychosocial resources for parents who may feel isolated or have concerns about competing priorities (e.g., employment, children at home). By leveraging the expertise of researchers, family engagement, and an emerging evidence base demonstrating the impact of visitation policies, it is possible to create an inpatient experience that preserves the ethical, legal, and public health principles of minimizing the spread of COVID-19 pandemic while continuing to conduct high-impact research, promote the FCC approach essential for quality pediatric care, and mitigate against health inequities.

\section{AUTHOR CONTRIBUTIONS}

All authors have made substantial contributions to conception and design, drafting the article or revising it critically for important intellectual content, and final approval of the version to be published.

\section{ADDITIONAL INFORMATION}

Competing interests: The authors declare no competing interests.

Publisher's note Springer Nature remains neutral with regard to jurisdictional claims in published maps and institutional affiliations.

\section{REFERENCES}

1. Goyal, M. K. et al. Racial and/or ethnic and socioeconomic disparities of SARSCoV-2 infection among children. Pediatrics 146, e2020009951 (2020).

2. Hart, J. L., Turnbull, A. E., Oppenheim, I. M. \& Courtright, K. R. Family-centered care during the COVID-19 era. J. Pain Symptom Manag. 60, e93-e97 (2020).

3. Virani, A. K. et al. Benefits and risks of visitor restrictions for hospitalized children during the COVID pandemic. Pediatrics 146, e2020000786 (2020).

4. Van Driest, S. L. et al. Research consent rates before and during a COVID-19 onevisitor policy in a children's hospital. Pediatr Res. 1-3. 2021. https://doi.org/ 10.1038/s41390-020-01303-7. Online ahead of print.

5. Weiner, D. L., Balasubramaniam, V., Shah, S. I. \& Javier, J. R., Pediatric Policy C. COVID-19 impact on research, lessons learned from COVID-19 research, implications for pediatric research. Pediatr. Res. 88, 148-150 (2020). 
Unintended consequences of restrictive visitation policies during the... JL Raphael et al.

6. Meert, K. L., Clark, J. \& Eggly, S. Family-centered care in the pediatric intensive care unit. Pediatr. Clin. N. Am. 60, 761-772 (2013).

7. Murray, P. D. \& Swanson, J. R. Visitation restrictions: is it right and how do we support families in the NICU during COVID-19? J. Perinatol. 40, 1576-1581 (2020).

8. Darcy Mahoney, A. et al. Impact of restrictions on parental presence in neonatal intensive care units related to coronavirus disease 2019. J. Perinatol. 40(Suppl. 1), 36-46 (2020).
9. Johnson, T. J. Intersection of bias, structural racism, and social determinants with health care inequities. Pediatrics. 146, e2020003657 (2020).

10. Weiner, H. S. et al. Hospital visitation policies during the SARS-CoV-2 pandemic. Am. J. Infect. Control. S0196-6553(20)30887-7 (2020). https://doi.org/10.1016/j. ajic.2020.09.007.

11. Zeh, R. D. et al. Impact of visitor restriction rules on the postoperative experience of COVID-19 negative patients undergoing surgery. Surgery 168, 770-776 (2020). 